\title{
Introduction
}

Journal of

Molecular Microbiology

Published online: July 9, 2015 and Biotechnology

DOI: $10.1159 / 000381215$

\section{The Bacterial Phosphotransferase System: New Frontiers 50 Years after Its Discovery}

\author{
Milton H. Saier Jr. \\ Department of Molecular Biology, University of California at San Diego, La Jolla, Calif., USA
}

\section{Key Words}

Phosphotransferase system · Enzyme II complex .

Escherichia coli $\cdot$ PTS50 symposium

\begin{abstract}
In 1964, Kundig, Ghosh and Roseman reported the discovery of the phosphoenolpyruvate:sugar phosphotransferase system (PTS), which they subsequently proposed might catalyze sugar transport as well as sugar phosphorylation. What we have learned in the 50 years since its discovery is that, in addition to these primary functions, the PTS serves as a complex protein kinase system that regulates a wide variety of transport, metabolic and mutagenic processes as well as the expression of numerous genes. Recent operon- and genome-sequencing projects have revealed novel PTS proteinencoding genes, many of which have yet to be functionally defined. The current picture of the PTS is that of a complex system with ramifications in all aspects of cellular physiology. Moreover, its mosaic evolutionary history is unusual and intriguing. The PTS can be considered to serve many prokaryotes in capacities of communication and coordination, as do the nervous systems of animals.
\end{abstract}

(c) 2015 S. Karger AG, Basel

\section{Introduction}

Fifty years ago, a novel sugar-phosphorylating system was discovered in Escherichia coli [Kundig et al., 1964]. The unique features of this phosphotransferase system (PTS) included the use of phosphoenolpyruvate (PEP) as the phosphoryl donor for sugar phosphorylation and the presence of three essential catalytic entities, termed Enzyme I, Enzyme II and HPr (heat-stable, histidinephosphorylatable protein). The discovery of this system provided an explanation for pleiotropic carbohydratenegative mutants of E. coli described as early as 1949 [Doudoroff et al., 1949].

As noted above, the three initially recognized activities of the E. coli PTS were presumed to correspond to three proteins. Now, several dozen PTS proteins are known in E. coli [Tchieu et al., 2001], with even more in some Firmicutes [Barabote and Saier, 2005; Comas et al., 2008]. More recently, genes encoding proteins of the PTS have been identified and characterized in Archaea [Cai et al., 2014; Pickl et al., 2012], but no eukaryote has yet been shown to possess a protein constituent of the system. It therefore appears to be a prokaryote-specific system, a potential target of antimicrobial agents.

\section{KARGER 125}

E-Mail karger@karger.com www.karger.com $/ \mathrm{mmb}$
(C) 2015 S. Karger AG, Base

$1464-1801 / 15 / 0253-0073 \$ 39.50 / 0$
Milton H. Saier Jr.

Department of Molecular Biology

University of California at San Diego

La Jolla, CA 92093-0116 (USA)

E-Mail msaier@ucsd.edu 


\section{Known PTS Functions}

In 1964, a single function for the PTS, namely sugar phosphorylation, was known. Fifty years later, we found that this system plays roles in many surprising aspects of bacterial physiology (table 1). Established primary functions of the system include sugar transport and phosphorylation as well as sugar reception for chemotactic responses, whereas secondary functions include various ramifications of metabolic and transcriptional regulation [Lengeler and Jahreis, 2009; Vastermark and Saier, 2014]. Targets of PTS-mediated regulation include: (i) carbohydrate catabolic enzymes, sugar permeases and the cyclic (c)AMP biosynthetic enzyme, adenylate cyclase, all regulated allosterically by the IIA ${ }^{\text {G1c }}$ PTS protein in enteric bacteria; (ii) a variety of transcription factors, non-PTS transport systems and even the PTS itself, regulated by $\operatorname{HPr}($ ser-P)-dependent allostery in many bacteria, including Gram-positive Firmicutes; (iii) transcriptional activators and antiterminators regulated by direct PTS-mediated phosphorylation in both Gramnegative and Gram-positive bacteria; (iv) other transcriptional regulators controlled indirectly by PTS-mediated phosphorylation by virtue of direct interactions with PTS proteins; (v) metabolism of nitrogen- and phosphorus-containing compounds; (vi) intracellular ionic $(\mathrm{K}+$ ) homeostasis; (vii) carbon (glycogen, $\beta$-hydroxybutyrate) storage; (viii) biofilm formation; (ix) virulence and $(\mathrm{x})$ directed transposon insertion for the purpose of operon activation (directed mutation) [Barabote and Saier, 2005; Krausse et al., 2009; Le Bouguenec and Schouler, 2011; Lopes et al., 2011; Pickering et al., 2014; Zhang and Saier, 2009a, b]. Moreover, the detection of novel, functionally uncharacterized PTS proteins in bacteria as diverse as E. coli, Acholeplasma laidlawii, Listeria monocytogenes and several antibioticproducing species of Streptomyces, suggests the involvement of PTS proteins in cellular processes distinct from those listed in table 1 [Barabote and Saier, 2005; Deutscher et al., 2014; Rigali et al., 2006].

\section{Historical Background}

In his first insightful review on the PTS, Saul Roseman, who died in 2011 at the age of 90, noted a potential analogy between the PTS-catalyzed coupled transport and phosphorylation of sugars and Peter Mitchell's postulated mechanism of group translocation, a process in which penetration of the membrane by a solute occurs
Table 1. Primary (metabolic) and secondary (regulatory) functions currently recognized for the PTS

\author{
Primary metabolic functions \\ Catalysis of: \\ Sugar transport \\ Sugar phosphorylation \\ Sugar chemoreception \\ Secondary regulatory functions \\ Control of the activities of: \\ PTS permeases \\ Non-PTS permeases \\ Catabolic enzymes \\ Adenylate cyclase \\ Transcription factors (many) \\ Control of physiological processes including: \\ Ionic homeostasis (potassium transport) \\ Glycogen accumulation \\ Polyhydroxybutyrate storage \\ Nitrogen utilization \\ Phosphorus metabolism \\ Biofilm formation \\ Virulence \\ Transposon-mediated directed mutation
}

concomitantly with a chemical reaction that modifies the transported substrate [Mitchell, 1954; Roseman, 1969]. Evidence for the involvement of the PTS in sugar transport had been published in 1966 [Kundig et al., 1966]. Although the experimental design utilized in the reported studies proved to be both conceptually and technically faulty, the conclusion that the PTS is a transport system proved to be correct [Postma et al., 1993]. Thus, in this instance, intuition provided a more accurate view of reality than did empiricism.

The first PTS permease to be successfully purified [Jacobson et al., 1983, 1979], reconstituted in an artificial membrane [Leonard and Saier, 1983] and sequenced [Lee and Saier, 1983] was the mannitol Enzyme II of E. coli. Since then, several other PTS permeases have been purified and characterized and, recently, the high-resolution 3D structure of an integral membrane PTS Enzyme IIC was determined [Cao et al., 2011; McCoy et al., 2015]. The high-resolution structures of several IIA and IIB proteins (see below) have also been solved, alone or complexed to other PTS or non-PTS proteins. These basic scientific breakthroughs allow us to understand the PTS-mediated phosphoryl transfer and transport mechanisms in structural detail [McCoy et al., 2015]. DOI: $10.1159 / 000381215$
Saier Jr. 


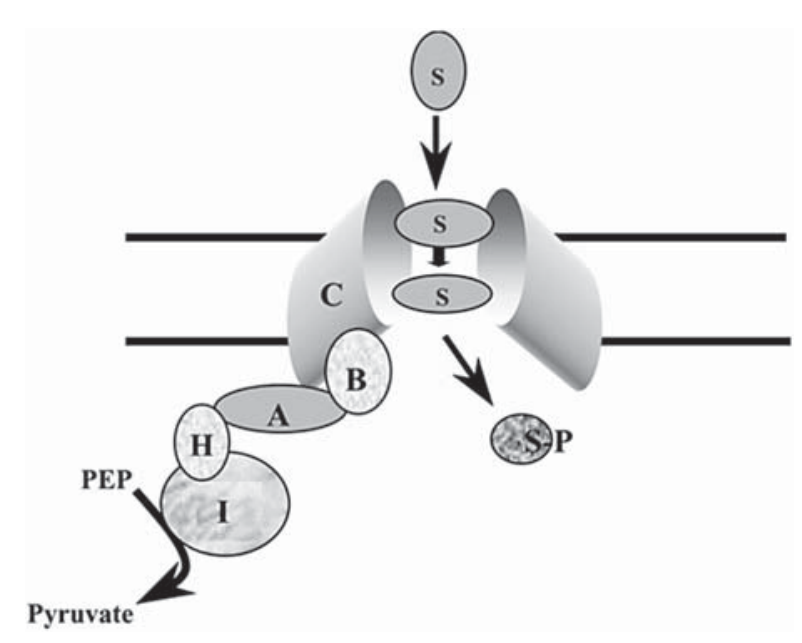

Fig. 1. Schematic depiction of the protein constituents of a typical PTS permease. A PTS permease is a sugar-transporting Enzyme II complex of the bacterial PEP-dependent phosphotransferase system. The sugar substrate $(S)$ is transported from the extracellular medium through the membrane in a pathway determined by the integral membrane permease-like Enzyme IIC (C) constituent, a homodimer in the membrane as shown. The sequentially acting energy-coupling proteins transfer a phosphoryl group from the initial phosphoryl donor, PEP, to the ultimate phosphoryl acceptor, sugar, yielding a sugar-phosphate (S-P). These enzymes are: Enzyme I (I), $\operatorname{HPr}(\mathrm{H})$, Enzyme IIA (A) and Enzyme IIB (B). I is the first general energy-coupling protein; $\mathrm{H}$ is the second general energy-coupling protein; $\mathrm{A}$ is the family-specific phosphoryl donor; $\mathrm{B}$ is the permease-specific phosphoryl donor, and $\mathrm{C}$ is the permease/receptor that mediates transport and catalyzes concomitant phosphorylation of the sugar substrate. A given bacterial cell may possess multiple PTS Enzyme II complexes, each specific for a different sugar or set of sugars. Some bacteria also possess multiple sets of PTS energy-coupling proteins (Enzymes I, HPr, IIA and IIB) that may play regulatory roles dependently or independently of sugar transport.

\section{PTS Structure and Function}

PTS permeases consist of two peripheral cytoplasmic membrane proteins or domains (IIA and IIB; sugar-specific energy coupling proteins) as well as one (or sometimes two) integral membrane proteins or domains (IIC, the actual sugar permease, with or without IID, depending on the system; fig. 1; table 2). These protein domains can be detached or fused together so that the Enzyme II complex can consist of one, two, three or four polypeptide chains encoded by the same number of genes. The IIA, IIB and IIC domains are frequently covalently linked to each other via gene-encoded flexible peptide linkers in a variable but nonrandom fashion. Thus, for example, a PTS permease
Table 2. Four evolutionarily distinct families of PTS Enzyme IIC proteins and their characteristics

The Glc-Fru-Lac superfamily

- Fru has been proposed to be the original PTS, but other IIC proteins diverged in function, sequence and possibly structure

- A basic dimeric 10 TMS topology, with two functionally different 5TMS halves per promoter is established for one member, but topological variations for other family members are possible

- IIAs and IIBs have mosaic origins, e.g. IIA ${ }^{\text {Glc }}$ is not homologous to IIA ${ }^{\mathrm{Ntr}}$ or IIA ${ }^{\mathrm{Lac}}$, and IIB ${ }^{\mathrm{Glc}}$ is not homologous to $\mathrm{IIB}^{\mathrm{Chb}}$

- IIAs, IIBs and IICs did not coevolve

The Asc-Gat superfamily

- IIC $^{\text {Asc }}$ homologues are often fused to IIA and IIB homologues, but IIC ${ }^{\text {Gat }}$ homologues never are. The IIC proteins have an 11 or 12 TMS topology, probably with a basic internal 5 TMS repeat unit

- IIC ${ }^{\text {Asc }}$ homologues are always encoded by genes in operons with IIA and IIB genes, but IIC ${ }^{\text {Gat }}$ homologues can be encoded in operons lacking IIA and IIB genes

- Some IICGat homologues are found in organisms that lack all other PTS proteins, suggesting roles as secondary carriers

- Asc and Gat IIA and IIB constituents are distantly related to IIA and IIB constituents of the Glc-Fru-Lac superfamily

The Man family

- All constituents (IIA, IIB, IIC and IID) differ structurally from all other PTS permease proteins

- All members, but only members of this family, have IID constituents

- The IIB constituents are phosphorylated on His rather than Cys residues as is true for other IIB proteins

- Members often exhibit broad specificity for aldo and keto sugars

The Dha family

- DhaK and DhaL are homologous to the N- and C-terminal domains of ATP-dependent DHA kinases

- DhaM consists of three domains: IIA ${ }^{\text {Man }}$-HPr-I $\Delta$

- The three domains of DhaM are phosphorylated by PEP, EI and $\mathrm{HPr}$, but DhaK and $\mathrm{L}$ are not phosphorylated

- DhaK binds DHA covalently to a His residue and transfers the phosphoryl group from IIA of DhaM via DhaL-ADP to DHA

I $\Delta$ is a truncated (partially deleted) Enzyme I. See text and figure 1 for protein designations. Glc $=$ Glucose; Fru = fructose; $\mathrm{Lac}=$ lactose $; \mathrm{Asc}=1$ - ascorbate $; \mathrm{Gat}=$ galactitol $;$ Man = mannose; $\mathrm{Chb}=$ diacetylchitobiose; Dha or DHA = dihydroxyacetone. 
containing a IIC domain may have the corresponding IIB domain either preceding or following it, but the IIA domain, when present in the same polypeptide chain, is usually linked at the $C$-terminal end of the permease.

The linking of IIA domains to general or sugar-inducible energy-coupling protein domains can occur either $\mathrm{N}$ - or $\mathrm{C}$-terminal to the latter proteins. In a particular PTS permease, a domain may be either deleted, as is true of the IIA domains of the sucrose and trehalose permeases of $E$. coli, duplicated, as is true of the IIB domains of the fructose permeases of E. coli and Rhodobacter capsulatus [Wu et al., 1990], or spliced in half, as seems to be true of the IIC domain of the glucitol permease of E. coli [Yamada and Saier, 1987]. If a IIA domain is deleted, then another, such as IIA ${ }^{\text {Glc }}$, must substitute for it since IIA, IIB and IIC are all required for PTS-mediated sugar phosphorylation.

\section{The Mosaic Origins of PTS Permeases (Enzyme II Complexes)}

PTS Enzyme IIC proteins/domains fall into four families with members of each family being homologous, deriving from a common ancestral protein (table 2; see subclass 4.A in the Transporter Classification Database, TCDB; www.tcdb.org) [Saier et al., 2006, 2009, 2014]. The first of these four families is the Glucose-Fructose-Lactose (Glc-Fru-Lac) family, the largest of the four families. One member of this family, the diacetylchitobiose IIC protein, is now known to have a basic 10 transmembrane a-helical segment (TMS) topology consisting of two 5 TMS domains serving different functions [Cao et al., 2011; McCoy et al., 2015]. The split glucitol IIC system is probably a distant member of this superfamily. The second family, the Ascorbate-Galactitol (Asc-Gat) family, has members with 11 or 12 TMSs with a primordial internal duplication of 5 TMSs. The evidence summarized in table 2 suggests that these IIC proteins may derive from secondary carriers, and some may still be capable of catalyzing transport in the absence of sugar phosphorylation, although this possibility has not been adequately tested [Hvorup et al., 2003; Saier et al., 2005]. The third family, the Mannose (Man) family, with IIC domains probably of 6 TMSs, as well as a second integral membrane constituent unique to this family (IID), appears to have evolved four protein constituents (IIA, IIB, IIC and IID), all of which arose independently of all other PTS proteins [Erni, 2006; Garcia-Alles et al., 2002]. Finally, the Dihydroxyacetone (Dha) family apparently derived relatively recently from a cytoplasmic primordial ATP-dependent dihydroxyacetone kinase [Bächler et al., 2005; Erni et al., 2006; Saier et al., 2005] (table 2).

As emphasized in table 2, the IIA and IIB PTS proteins did not coevolve with the IIC constituents, and it is possible that different IIC members of the Glc-Fru-Lac family actually have different topologies [McCoy et al., 2015]. These observations reveal that the PTS is truly a mosaic system, derived from many ancestral sources that probably evolved independently of each other and later came together to form functional Enzyme II complexes [Saier and Reizer, 1994; Saier et al., 2005]. Thus, the PTS may have evolved late, after the appearance of established universal metabolic pathways such as glycolysis, gluconeogenesis and the Krebs cycle.

\section{Overview of This PTS50 Written Symposium}

The PTS scientific community celebrated the 50th anniversary of the discovery of the PTS in the summer of 2014 in Göttingen, Germany. The meeting was organized by Jörg Stülke, Josef Deutscher and myself. Over 100 people, most of them actively researching the PTS, attended the meeting, and many of them presented talks or posters describing their recent research efforts. All in attendance agreed it was a most informative and enjoyable event with old friends meeting new members of the PTS community while exchanging scientific and personal information.

The first session of the meeting was opened by Joseph Lengeler of Germany who presented a historical view of the PTS in a talk which formed the basis for the first fulllength article in this Journal of Molecular Microbiology and Biotechnology written symposium. The second article in the symposium, by Joyet et al., describes the recent research conducted within the research group of Josef Deutscher in France. This article concerns the involvement of the PTS in transcriptional regulation in Grampositive Firmicutes. The next three articles in this symposium deal with different aspects of the PTS in Firmicutes, the first by Van der Heiden et al. (Belgium), dealing with the newly characterized PTS-mediated D-tagatose catabolic pathway in Bacillus licheniformis, the second, by Kuipers et al. (The Netherlands), concerning PTS-mediated fucose (6-deoxygalactose) metabolism in the pathogen Streptococcus pneumoniae, and the third, by W.J. Mitchell (UK), dealing with genomic analyses of the PTS in solventogenic Clostridia, fairly close relatives of the Bacilli. 
The sixth symposium article, by Gordon et al., from the research group of Orna Amster-Chloder in Israel, deals with the bioinformatic identification and functional characterization of PTS-regulated RNA antiterminator-like sites in bacterial genomes, a paper that suggests the occurrence of novel functions for these sites. In the seventh article, Jacqueline Plumbridge (France) contrasts the regulation of amino sugar utilization by the proteobacterium E. coli and the firmicute B. subtilis. Two subsequent papers, one by Luettman et al. from Boris Görke's laboratory (Austria), and the second by Wolf et al. from Andreas Kremling's laboratory (Germany), deal with the involvement of the nitrogen regulatory PTS $\left(\mathrm{EI}^{\mathrm{Ntr}}, \mathrm{NPr}\right.$ and IIA $\left.{ }^{\mathrm{Ntr}}\right)$ in the control of potassium ion uptake in two distantly related $\gamma$-Proteobacteria, Pseudomonas putida and E. coli, respectively. These papers describe a novel function of the PTS in maintaining ionic homeostasis.

In the tenth article, Carmona et al. (Guillermo Gosset's laboratory in Mexico) discuss the use of pts mutants of $E$. coli to facilitate the industrial production of various compounds, including aromatic metabolites and biofuels. As also noted in Wilf Mitchell's article, the biotechnological use of bacteria for useful metabolite production is of growing interest and utility. Sarah Sutrina of the West Indies, in the eleventh symposium article, summarizes the work executed in her laboratory defining the characteristics of $E$. coli biofilms and how these 'differentiated' multicellular structures are organized and regulated during their biogenesis by the PTS and cAMP.

The final article, by my research associate, Zhongge Zhang and myself (USA), deals with the novel and surprising observation that the PTS regulates the process of directed mutation of the glycerol catabolic operon, $g l p F K$, in E. coli. The gene products of this operon are essential for the normal growth of these bacteria with this compound as the sole carbon source. The PTS controls cAMP synthesis and inducer uptake, as established in previous publications from several laboratories. In this case, glycerol uptake via the glycerol facilitator, GlpF, is followed by its glycerol kinase (GlpK)-dependent phosphorylation to glycerol 3-phosphate, the true inducer of the glycerol ( $g l p)$ utilization regulon. These two small molecules, cAMP and glycerol 3-P, interact with their cognate transcription factors, the cAMP receptor protein (Crp), an activator of transcription, and the glycerol repressor (GlpR), a negative effector of transcription, respectively. Binding of either of these two proteins to their DNA binding sites in the $g l p F K$ operon inhibits, and thereby regulates, insertion of a small transposon, IS5, into a sin- gle upstream site of the $g l p F K$ promoter, thereby activating this promoter and allowing the bacteria to overcome the inhibitory effects of nonmetabolizable PTS sugars on glycerol utilization [Saier and Zhang, 2014; Zhang and Saier, 2009b, a]. This novel discovery represents the most recently identified regulatory function of the PTS.

\section{Concluding Remarks}

The studies reported in this comprehensive PTS50 written symposium illustrate the fact that our understanding of the PTS is still expanding with respect to its structure, mechanism, evolution and physiological functions. Since the last 50 years of PTS research have been so revealing, we wonder what the next 50 years will yield. Structural diversity in the proteins that comprise the system, novel mechanisms of catalysis and undreamt of functions are likely to come to light. Only a few of us may live to see that day. Nevertheless, our collective efforts will hopefully live on if we humans do not destroy the only habitat we have, the planet Earth.

\section{Acknowledgements}

I thank the many participants of the PTS50 congress for their insightful oral and written contributions and discussions. Jörg Stülke and Josef Deutscher were instrumental in ensuring the smooth flow of events at the meeting, making it most memorable for the PTS research community. Research in the Saier laboratory was supported by NIH grants GM077402, GM109859 and GM094610.

References
Bahler P, Erni B: From ATP as substrate to
ADP as coenzyme: functional evolution of the
nucleotide binding subunit of dihydroxyace-
tone kinases. J Biol Chem 2005;280:18321-
18325 .
Barabote RD, Saier MH Jr: Comparative genomic
analyses of the bacterial phosphotransferase
system. Microbiol Mol Biol Rev 2005;69:608-
634.
Cai L, Cai S, Zhao D, Wu J, Wang L, Liu X, Li M,
Hou J, Zhou J, Liu J, Han J, Xiang H: Analysis
of the transcriptional regulator GlpR, pro-
moter elements, and posttranscriptional pro-
cessing involved in fructose-induced activa-
tion of the phosphoenolpyruvate-dependent
sugar phosphotransferase system in Halofe-
rax mediterranei. Appl Environ Microbiol
2014;80:1430-1440.
77
The Bacterial PTS: New Frontiers 50

Years after Its Discovery
J Mol Microbiol Biotechnol 2015;25:73-78 DOI: $10.1159 / 000381215$ 
Cao Y, Jin X, Huang H, Derebe MG, Levin EJ, Kabaleeswaran V, Pan Y, Punta M, Love J, Weng J, Quick M, Ye S, Kloss B, Bruni R, MartinezHackert E, Hendrickson WA, Rost B, Javitch JA, Rajashankar KR, Jiang Y, Zhou M: Crystal structure of a potassium ion transporter, TrkH. Nature 2011;471:336-340.

-Comas I, Gonzalez-Candelas F, Zuniga M: Unraveling the evolutionary history of the phosphoryl-transfer chain of the phosphoenolpyruvate:phosphotransferase system through phylogenetic analyses and genome context. BMC Evol Biol 2008;8:147.

Deutscher J, Ake FM, Derkaoui M, Zebre AC, Cao TN, Bouraoui H, Kentache T, Mokhtari A, Milohanic E, Joyet P: The bacterial phosphoe nolpyruvate:carbohydrate phosphotransferase system: regulation by protein phosphorylation and phosphorylation-dependent protein-protein interactions. Microbiol Mol Biol Rev 2014;78:231-256.

Doudoroff M, Hassid WZ, et al: Direct utilization of maltose by Escherichia coli. J Biol Chem 1949;179:921-934.

-Erni B: The mannose transporter complex: an open door for the macromolecular invasion of bacteria. J Bacteriol 2006;188:7036-7038.

-Erni B, Siebold C, Christen S, Srinivas A, Oberholzer A, Baumann U: Small substrate, big surprise: fold, function and phylogeny of dihydroxyacetone kinases. Cell Mol Life Sci 2006;63:890-900

Garcia-Alles LF, Zahn A, Erni B: Sugar recognition by the glucose and mannose permeases of Escherichia coli: steady-state kinetics and inhibition studies. Biochemistry 2002;41: 10077-10086.

Hvorup R, Chang AB, Saier MH Jr: Bioinformatic analyses of the bacterial L-ascorbate phosphotransferase system permease family. J Mol Microbiol Biotechnol 2003;6:191-205.

-Jacobson GR, Lee CA, Leonard JE, Saier MH Jr: Mannitol-specific Enzyme II of the bacterial phosphotransferase system. I. Properties of the purified permease. J Biol Chem 1983;258: 10748-10756.

-Jacobson GR, Lee CA, Saier MH Jr: Purification of the mannitol-specific Enzyme II of the Escherichia coli phosphoenolpyruvate:sugar phosphotransferase system. J Biol Chem 1979;254:249-252.

-Krausse D, Hunold K, Kusian B, Lenz O, Stulke J, Bowien B, Deutscher J: Essential role of the $h p r K$ gene in Ralstonia eutropha H16. J Mol Microbiol Biotechnol 2009;17:146-152.
Kundig W, Ghosh S, Roseman S: Phosphate bound to histidine in a protein as an intermediate in a novel phospho-transferase system. Proc Natl Acad Sci USA 1964;52:1067-1074.

Kundig W, Kundig FD, Anderson B, Roseman S: Restoration of active transport of glycosides in Escherichia coli by a component of a phosphotransferase system. J Biol Chem 1966;241: 3243-3246.

Le Bouguenec C, Schouler C: Sugar metabolism, an additional virulence factor in enterobacteria. Int J Med Microbiol 2011;301:1-6.

Lee CA, Saier MH Jr: Mannitol-specific Enzyme II of the bacterial phosphotransferase system. III. The nucleotide sequence of the permease gene. J Biol Chem 1983;258:10761-10767.

Lengeler JW, Jahreis K: Bacterial PEP-dependent carbohydrate: phosphotransferase systems couple sensing and global control mechanisms. Contrib Microbiol 2009;16:65-87.

Leonard JE, Saier MH Jr: Mannitol-specific Enzyme II of the bacterial phosphotransferase system. II. Reconstitution of vectorial transphosphorylation in phospholipid vesicles. Biol Chem 1983;258:10757-10760.

Lopes MS, Gosset G, Rocha RC, Gomez JG, Ferreira da Silva L: PHB biosynthesis in catabolite repression mutant of Burkholderia sacchari. Curr Microbiol 2011;63:319-326.

McCoy JG, Levin EJ, Zhou M: Structural insight into the PTS sugar transporter EIIC. Biochim Biophys Acta 2015;1850:577-585.

Mitchell P: Transport of phosphate across the osmotic barrier of Micrococcus pyogenes: specificity and kinetics. J Gen Microbiol 1954;11: 73-82.

Pickering BS, Lopilato JE, Smith DR, Watnick PI: The transcription factor Mlc promotes Vibrio cholerae biofilm formation through repression of phosphotransferase system components. J Bacteriol 2014;196:2423-2430.

Pickl A, Johnsen U, Schonheit P: Fructose degradation in the haloarchaeon Haloferax volcanit involves a bacterial type phosphoenolpyruvate-dependent phosphotransferase system, fructose-1-phosphate kinase, and class II fructose-1,6-bisphosphate aldolase. J Bacteriol 2012;194:3088-3097.

Postma PW, Lengeler JW, Jacobson GR: Phosphoenolpyruvate:carbohydrate phosphotransferase systems of bacteria. Microbiol Rev 1993; 57:543-594.

Rigali S, Nothaft H, Noens EE, Schlicht M, Colson S, Muller M, Joris B, Koerten HK, Hopwood DA, Titgemeyer F, van Wezel GP: The sugar phosphotransferase system of Streptomyces coelicolor is regulated by the GntR-family regulator DasR and links $\mathrm{N}$-acetylglucosamine metabolism to the control of development. Mol Microbiol 2006;61:1237-1251.

Roseman S: The transport of carbohydrates by a bacterial phosphotransferase system. J Gen Physiol 1969;54:138-184.

- Saier MH, Hvorup RN, Barabote RD: Evolution of the bacterial phosphotransferase system: from carriers and enzymes to group translocators. Biochem Soc Trans 2005;33:220-224.

- Saier MH Jr, Reddy VS, Tamang DG, Vastermark A: The transporter classification database. Nucleic Acids Res 2014;42:D251-D258.

- Saier MH Jr, Reizer J: The bacterial phosphotransferase system: new frontiers 30 years later. Mol Microbiol 1994;13:755-764.

- Saier MH Jr, Tran CV, Barabote RD: TCDB: the Transporter Classification Database for membrane transport protein analyses and information. Nucleic Acids Res 2006;34:D181D186.

- Saier MH Jr, Yen MR, Noto K, Tamang DG, Elkan C: The transporter classification database: recent advances. Nucleic Acids Res 2009; 37:D274-D278.

Saier MH Jr, Zhang Z: Transposon-mediated directed mutation controlled by DNA binding proteins in Escherichia coli. Front Microbiol 2014;5:390.

Tchieu JH, Norris V, Edwards JS, Saier MH Jr: The complete phosphotransferase system in Escherichia coli. J Mol Microbiol Biotechnol 2001;3:329-346.

-Vastermark A, Saier MH Jr: The involvement of transport proteins in transcriptional and metabolic regulation. Curr Opin Microbiol 2014; 18:8-15.

Wu LF, Tomich JM, Saier MH Jr: Structure and evolution of a multidomain multiphosphoryl transfer protein: nucleotide sequence of the fruB(HI) gene in Rhodobacter capsulatus and comparisons with homologous genes from other organisms. J Mol Biol 1990;213:687-703.

- Yamada M, Saier MH Jr: Glucitol-specific enzymes of the phosphotransferase system in Escherichia coli: nucleotide sequence of the gut operon. J Biol Chem 1987;262:5455-5463.

Zhang Z, Saier MH Jr: A novel mechanism of transposon-mediated gene activation. PLoS Genet 2009a;5:e1000689.

Zhang Z, Saier MH Jr: A mechanism of transposon-mediated directed mutation. Mol Microbiol 2009b;74:29-43. 